\title{
Human brain responses associated with subjective evaluation of error significance are sensitive to error inevitability
}

Running head: Significance of avoidable errors

\section{Magdalena Senderecka ${ }^{a, *}$ and Jakub Szewczyk ${ }^{\text {b,c }}$}

a Institute of Philosophy, Jagiellonian University, Kraków, Poland

${ }^{\mathrm{b}}$ Department of Psychology, University of Illinois, Urbana-Champaign, United States of America

${ }^{\mathrm{c}}$ Institute of Psychology, Jagiellonian University, Kraków, Poland

\section{* Corresponding Author:}

Jagiellonian University, Institute of Philosophy, Grodzka 52, 31-044 Kraków, Poland E-mail address: magdalena.senderecka@uj.edu.pl (M. Senderecka) 
Significance of avoidable errors

\begin{abstract}
This study examined whether error-related brain responses are sensitive to the degree of error inevitability, a factor which seriously affects the attribution of moral responsibility for an error. We were especially interested in error-related negativity (ERN), which is an electrophysiological marker of subjective evaluation of error significance and its motivational value. In addition, we focused on post-error slowing, which is a post-error behavioural adjustment. We hypothesized that the more avoidable the error, and consequently the greater its significance and motivational value, the larger the ERN amplitude, the shorter the ERN latency, and the greater the post-error slowing should be. To elicit errors whose inevitability varies, we used the stop-signal task. The inevitability of errors in this task depends on whether the stop signal is presented before or after a point beyond which the completion of the movement cannot be cancelled. Consistent with our hypotheses, we found that the higher motivational value of avoidable errors was indeed reflected in larger and earlier ERNs. Moreover, avoidable errors led to greater adjustments in subsequent behaviour aimed at preventing similar failures in the future. These findings show that early performance monitoring, as reflected by ERN, involves an evaluation of error inevitability. In a broader perspective, these results indicate that the elementary basis for distinguishing between culpable (avoidable) and non-culpable (unavoidable) errors may occur in the brain several dozen milliseconds after error commission.
\end{abstract}

\title{
Keywords:
}

error-related negativity; event-related potentials; performance monitoring; post-error slowing; stop-signal task 
Significance of avoidable errors

\section{Introduction}

A widely accepted moral principle affirms that an agent is responsible for performing a given act only if he could have avoided doing it (Widerker \& McKenna, 2006). Let us imagine the following two hypothetical scenarios. A car is traveling along a city road at a moderate speed. In the first scenario, a pedestrian suddenly runs into the street just in front of the car. Although the driver brakes immediately, he fails to avoid a collision. In the second scenario, a pedestrian unexpectedly enters the street over a dozen meters in front of the car. Although the driver has enough space and time to stop the car without a collision, he applies the brakes too slowly, thus the vehicle hits the pedestrian. The damage in the first scenario is inevitable: nothing can be done to avoid it because driver has too little time to slow down and cannot avoid the crash. In contrast, the damage in the second scenario could have been avoided only if the driver had reacted faster. In both cases, the damage caused by the driver is similar, but in the second case we find it much more justified to blame him for the accident.

Usually, we can correctly differentiate between these two types of situations. Free behaviour requires alternate possibilities, thus there is no reason to hold an agent responsible for an act that is explainable solely in terms of deterministic causes. However, the question arises whether the brain also differentiates between these two types of errors: those that are avoidable and those that cannot be prevented. Are neural responses sensitive to the motivational value of errors that are avoidable to a greater or lesser extent? The goal of our study was to address this question by means of event-related potentials (ERPs) extracted from electroencephalograms (EEG).

Error processing is best reflected on the neural level by an event-related potential component called error-related negativity (ERN; Gehring et al., 1993), or error negativity (Ne; Falkenstein et al., 1990). ERN is a sharp negative wave that peaks over fronto-central brain areas around 0-100 ms after an erroneous response and is generated in the cingulate and 
medial-frontal cortex (Ridderinkhof et al., 2004). Several findings suggest that ERN reflects a mechanism that plays a major role in the evaluation of error significance. The ERN amplitude monotonically increases when the accuracy of response decreases, revealing parametric modulation of the monitoring system as a function of the severity of errors (Vocat et al., 2011; see also Bernstein et al., 1995; Luu et al., 2000). Thus, ERN amplitude is not simply an all-ornothing index of action failure: in fact, it encodes graded information about the perceived severity of such failures. Importantly for the present study, the more severe the error, the larger the ERN. ERN amplitude is also associated with the motivational significance of errors for ongoing behaviour and the level of the perceived responsibility for such failures. In particular, it has been observed that more pronounced ERNs co-occur with errors that have higher motivational value (Hajcak et al., 2004, 2005; Maier et al., 2008) and more internal attribution of responsibility (Rigoni et al., 2015). Thus, as a complement to previous findings, we expected that ERN amplitude should also exhibit sensitivity to the degree to which errors are avoidable. Since avoidable errors are subjectively more severe and have higher motivational value than those that cannot be avoided, we predicted that they should result in more pronounced ERN amplitude.

As for the latency, previous findings suggest that the time course of the ERN component covaries with error correction. In a study on overt errors by Fiehler, Ullsperger and Von Cramon (2005), participants were asked to perform a flanker task and immediately correct all encountered errors. The researchers observed that the longer the ERN latency, the longer it took participants to correct their errors. In turn, Ficarella Rochet and Burle (2019) found that the ERN response elicited by overt errors in the Simon task peaked later than ERN elicited by partial errors; namely, covert activations of muscles involved in incorrect response production, which briefly occurs in the electromyographic recording before the correct response is given, but does not manifest on the behavioural level (for similar results in the 
flanker task, see Roger et al., 2014). Importantly, these observations indicate that the sooner the corrective action begins, the shorter the ERN latency is. Based on previous results, we expected that ERN latency may differ not only between quickly and slowly corrected overt errors or between partial and overt errors, but also within errors that vary with respect to their inevitability. We assumed that in the case of avoidable errors, corrective processes (although ultimately ineffective) have enough time to begin and develop to certain extent before the incorrect response is given. Thus, such errors should produce short-latency ERNs.

The significance and motivational value of errors may also be inferred from post-error behavioural adjustments, which are typically implemented in order to reduce subsequent errors (for a review, see Danielmeier \& Ullsperger, 2011). The most important behavioural adjustment in the context of the present study is post-error slowing, which is defined as an increase in response time in trials directly following an error (Laming, 1979; Rabbitt, 1966). The more significant errors that are associated with larger ERNs evoke increased post-error slowing (Debener et al., 2005; Holroyd et al., 2005; Yeung et al. 2004). Thus, in parallel to changes in the amplitude and latency of the ERN, we expected that increased significance of avoidable errors should result in greater slowing of subsequent motor reactions aimed at preventing further errors.

The most suitable paradigms for investigating error processing are speeded-response tasks in which two different response options are activated simultaneously (as in the flanker or Simon tasks) or in quick succession (as in the stop-signal task). In this study we chose the latter paradigm because it provides a unique opportunity to trigger errors that vary in the degree to which they can be avoided (for an illustration, see Fig. 1). On each trial in the stopsignal task (Logan \& Cowan, 1984), participants are asked to make a simple decision as quickly as possible; for example, press a key corresponding to the direction of an arrow displayed on the screen. In a minority of trials, a stop signal is presented unexpectedly at 
different times after the go response is triggered but before the participants actually reacts. In such cases, the participant is asked to withhold the reaction. If the stop signal occurs after the so-called point of no return (cf., De Jong et al., 1990; Schultze-Kraft et al., 2016), the error is unavoidable; this is like the first scenario, when the driver cannot avoid a collision since a pedestrian steps into the road just in front of the car. In contrast, when the stop signal is presented before the point of no return, action correction (inhibition of response) is more probable, but errors may also occur; this is like the second scenario, when the driver perceives the pedestrian in advance but due to deficient action monitoring s/he fails to prevent the collision.

In the present study, we used the stop-signal task to determine whether error-related brain activity and post-error behavioural responses differentiate between failures that are describable solely in terms of deterministic variables (which implies external attribution of responsibility), and failures that are due to momentary deficits of intentional control (which, in contrast, implies internal attribution of responsibility). The longer the distance between the stop signal and the actual (erroneous) reaction of the participant, the more the error is perceived as avoidable. We asked if the increased significance and higher motivational value of such errors, compared to erroneous responses given a shorter time after the appearance of the stop signal, would be reflected in the brain activity associated with subjective evaluation of errors. Based on previous findings, we hypothesized that the longer the stop-response interval (the more avoidable the error), the shorter the ERN latency and the larger the ERN amplitude. Moreover, if the inevitability of the error truly has a motivational component, errors that are perceived as more avoidable should lead to a greater adjustment in further behaviour, i.e. they should lead to increased post-error slowing. 


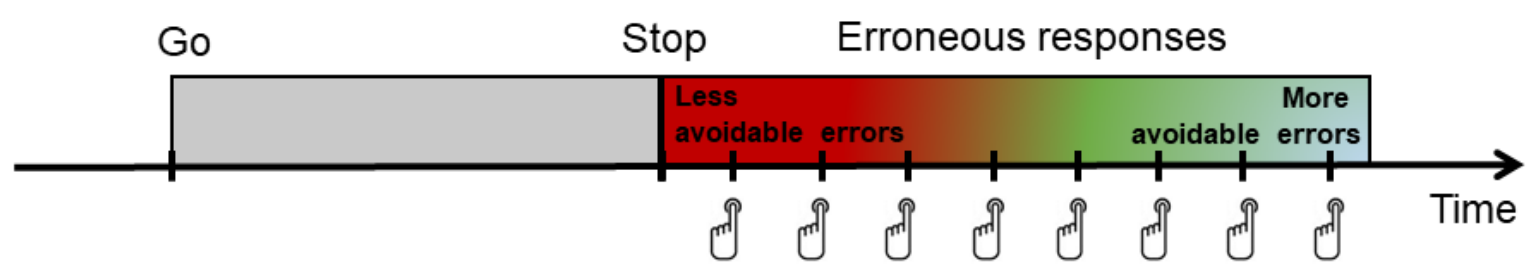

Fig. 1 - Errors avoidable to a greater or lesser extent in the stop-signal task. Erroneous responses given shortly after the stop-signal presentation are less avoidable than those given a longer time after the appearance of the stop-signal.

\section{Material and Methods}

\subsection{Participants}

Sixty-five volunteers (43 female and 22 male) aged $19-33$ years $(M=21.7$ years, $S D=2.6)$ were recruited from the general population via internet advertisements and through an online experimental recruitment system at the university. All participants were in good health, free of medications, and had normal or corrected-to-normal vision. None reported a history of psychiatric or neurological diseases. Of the initial sample recruited for the study, eight participants were excluded from the analyses due to probable misunderstanding of the instructions, thus leading to a lack or low number (six or less) of correctly inhibited responses; one participant was excluded because his mean RT deviated substantially from the mean of the sample (more than +3.0 standard deviations); another was excluded because of technical problems with the EEG recording. The remaining 55 participants ( 38 females and 17 males) were aged 19-27 years old, with a mean age of 21.5 years $(S D=2.1)$.

Since there are no prior studies that considered a similar research question, we could not obtain an estimate of the effect size necessary to perform a priori power analysis and determine the number of participants required to detect potential effects. To overcome this limitation, we determined the size of our sample based on a review of previous works on error processing, especially two studies (referenced in the Introduction) in which ERN amplitude or 
Significance of avoidable errors

latency were influenced by the magnitude or type of errors, respectively (Vocat et al., 2011; Ficarella et al., 2019). Compared to the previous studies, the sample of participants in our study was three times larger.

\subsection{Procedure and task}

The experimental procedure was in accordance with the ethical principles of the Declaration of Helsinki (World Medical Organization, 1996). The protocol was approved by the Research Ethics Committee at the Philosophical Faculty of Jagiellonian University in Kraków, Poland. All participants provided written informed consent prior to their participation. The whole experiment took place in a dimly lit, sound-attenuated, air-conditioned testing room. After filling out the questionnaires (the results of which have been described in Senderecka et al., 2018), participants completed the stop-signal task. This task required them to perform a primary binary-choice response task that included a visual go stimulus consisting of a small green square presented with the inner edge at two degrees either to the right or the left of the fixation point. The green square was presented randomly for $100 \mathrm{~ms}$ on the left or right side of a 23 " computer monitor (with $50 \%$ probability) on a black background, at eye-level, approximately $80 \mathrm{~cm}$ in front of the participant. Participants were instructed to indicate the location of the square by pressing the left or right "ctrl" key using their index fingers. Each trial began with the presentation of a white central fixation cross for $800 \mathrm{~ms}$, immediately followed by the presentation of the square.

In a random sample of $25 \%$ of the trials, a $1400 \mathrm{~Hz}$ tone which served as the stop signal was presented binaurally over Sennheiser HD 429 headphones (intensity 60 dB SPL, duration $100 \mathrm{~ms}$, rise and fall time $10 \mathrm{~ms}$ ). The sound prompted the participants to inhibit their responses to the primary go task, regardless of the location of the square. The interval between the presentation of the go stimulus and the stop signal (i.e. the stop-signal delay) was 
varied trial by trial using a tracking method: the interval increased or decreased by $50 \mathrm{~ms}$ (from 100 to $400 \mathrm{~ms}$ ) for the next stop-signal trial, depending on whether the participants successfully or unsuccessfully inhibited their response to the go stimulus. Thus, there were seven possible stop-signal delays: 100, 150, 200, 250, 300, 350, $400 \mathrm{~ms}$. The initial value of the stop-signal delay was set to $150 \mathrm{~ms}$. Fig. 2 presents an outline of the stop-signal task design.

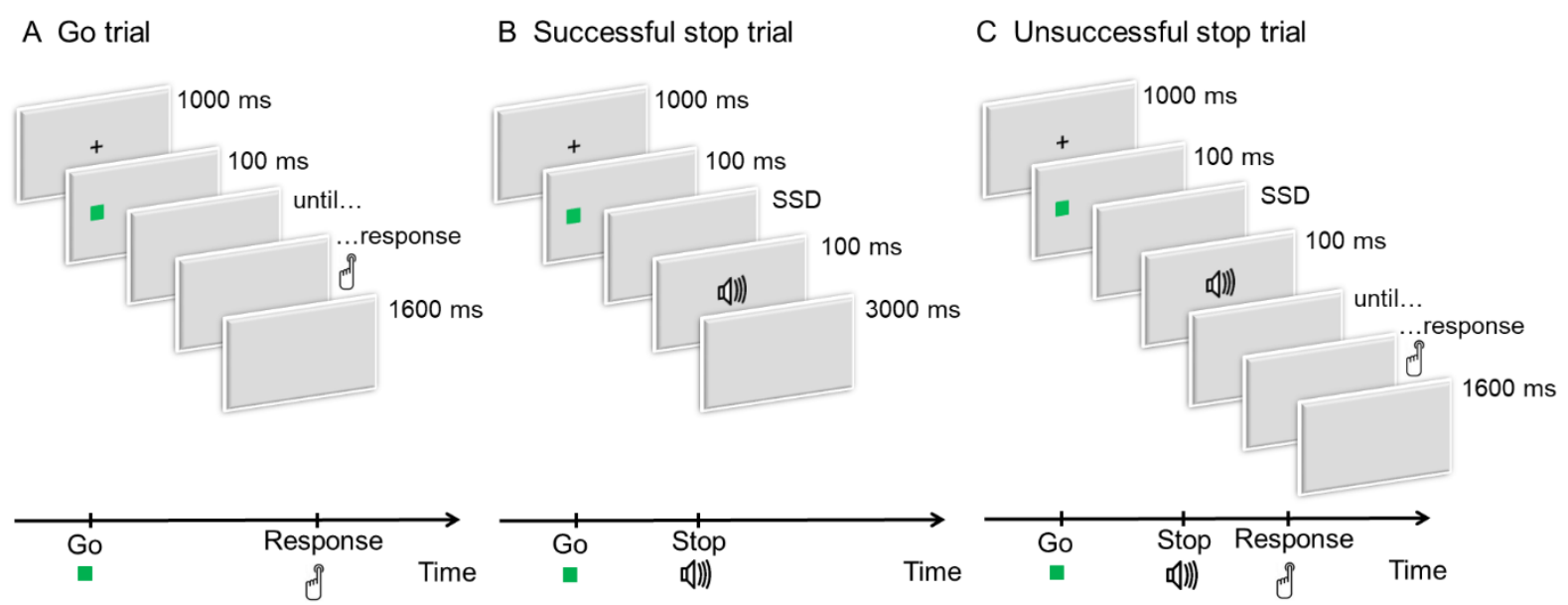

Fig. 2 - Stop-signal task. Each trial began with a fixation point in the middle of the screen for $1000 \mathrm{~ms}$ followed by a go stimulus. The go stimulus, namely a green square presented either to the right or the left of the fixation point, indicated that the participant should make a righthand or left-hand response, respectively. The go stimulus remained on the screen for $100 \mathrm{~ms}$. Twenty-five percent of trials contained the auditory stop signal, which followed the go stimulus and indicated that the participant should inhibit the given response. The stop-signal delay (SSD) between go and stop signals was adaptively adjusted. A blank screen occupied the remaining time between trials. Panel A presents a go trial without stop-signal presentation; panel B shows a successfully inhibited stop-signal trial; panel C illustrates an unsuccessfully inhibited stop-signal trial. 
Significance of avoidable errors

To ensure that they understood the task, participants performed one or two practice blocks of 24 trials before data collection. After the practice runs, they completed four experimental blocks, each consisting of 50 trials with short breaks in between. All participants were asked to restrict body movements and blinking as much as possible during the recording of the EEG. The task was implemented using DMDX software (Forster \& Forster, 2003).

\subsection{Electrophysiological recording and pre-processing}

The continuous scalp EEG signal was recorded from 32 silver/silver-chloride $(\mathrm{Ag} / \mathrm{AgCl})$ active electrodes (with pre-amplifiers) using the BioSemi ActiveTwo system. The electrodes were secured in an elastic cap (Electro Cap) according to the extended 10-20 international electrode placement system. The zero-reference principal voltage values (quantified relative to the driven right leg and common mode sense circuit) were digitized at a sampling rate of $256 \mathrm{~Hz}$. The horizontal and vertical electrooculograms were monitored using four additional electrodes placed above and below the right eye, and in the external canthi of both eyes.

All channels were re-referenced off-line to the average of the two mastoid electrodes. The recordings were filtered offline with a high-pass filter of $0.05 \mathrm{~Hz}$ (slope $24 \mathrm{~dB} / \mathrm{oct}$ ) and a low-pass filter of $25 \mathrm{~Hz}$ (slope $12 \mathrm{~dB} / \mathrm{oct}$ ). The EEG data were segmented relative to the go stimulus onset into -100-1900 ms segments. All trials were manually inspected to remove non-stationary artifacts (such as skin potentials or artifacts due to head movements). Ocular and other stationary artifacts were removed with the independent component analysis algorithm (ICA) using Brain Vision Analyzer 2 (Brain Products, Munich, Germany). ICA decomposition was applied to the EEG data using the Infomax Extended algorithm. Each participant's independent components were visually inspected. On the basis of the spatiotemporal pattern (Jung et al., 2000), components were removed that were recognized as blinks, saccades, participant's pulse, muscle artifacts, or a stationary artifact in a single 
electrode. Subsequently, contaminated trials exceeding maximum $/$ minimum amplitudes of \pm $65 \mu \mathrm{V}$ were rejected by a semi-automatic procedure. Artifact-free ERPs were aligned to the pre-go-stimulus baseline from $-100 \mathrm{~ms}$ to $0 \mathrm{~ms}$. Both unsuccessfully and successfully inhibited trials were retained.

Since stop signals and unsuccessfully inhibited (erroneous) responses occur in rapid succession, their ERPs can overlap temporally. The degree of overlap is partly a function of the stop-signal delay: the overlap is usually more evident in trials with a longer stop-signal delay, and it is less substantial in trials with a shorter stop-signal delay. To ensure that our results would not be confounded by differences in the degree of the overlap, we applied the following procedure (see De Jong et al., 1990; Kok et al., 2004; Ramautar et al., 2004; Schevernels et al., 2015 for similar examples). First, for each participant and stop-signal delay we averaged stop-signal-locked segments derived from successfully inhibited trials (i.e., without a motor response). As a result, for each stop-signal delay we obtained the most reliable estimation of the stop-signal-related wave that was not influenced by response-related activation. Second, from every single segment derived from unsuccessfully inhibited trials (i.e. stop trials with a motor response), we subtracted the previously averaged stop-signalrelated wave. Importantly, single segments and averaged waves were matched in terms of the stop-signal delay. In this way, we removed the go- and stop-processing overlap from unsuccessfully inhibited trials, which allowed us to examine processing specifically related to the erroneous motor response on the single-trial level.

As a result, we obtained 1,354 artifact-free single trials that were extracted from difference waves, time-locked to the erroneous response, and subsequently included in the ERP analysis. Following standard practice and according to the literature (Miltner et al., 2003), the ERN component was analysed at averaged $\mathrm{Fz}$ and $\mathrm{Cz}$ electrodes. To ensure that the whole spectrum of available ERN components with different time courses would be taken into 
account, the time window selection was based on the distribution of the single-trial ERN latencies, which is presented in Fig. 3. The majority of ERN peaks occurred between -50 and $300 \mathrm{~ms}$ around the response. Therefore, for the analysis of ERN amplitude we used the mean amplitude in this time window, whereas for the analysis of ERN latency we used the latency of the time point with the most negative amplitude within this time window.

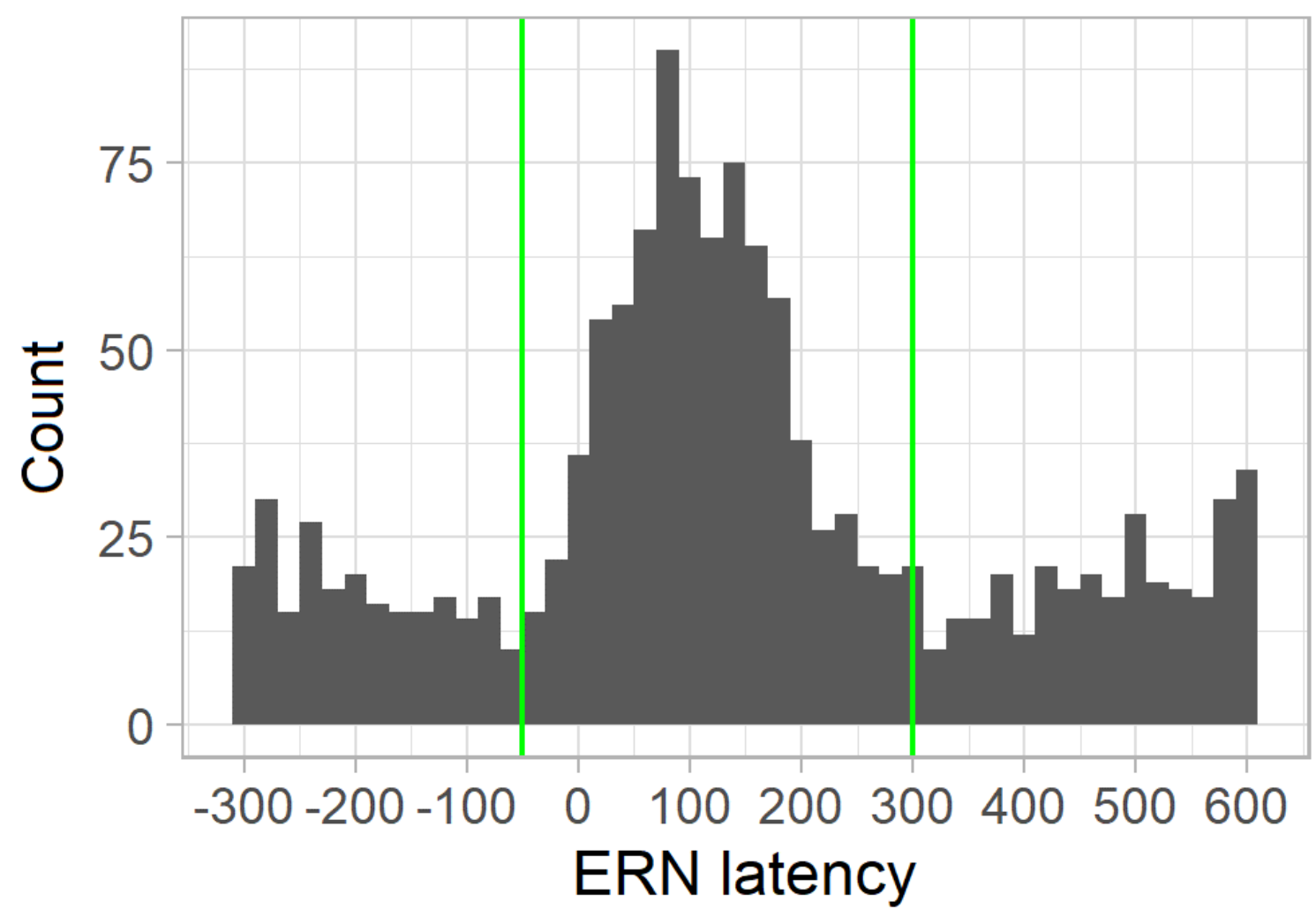

Fig. 3 - Distribution of the single-trial ERN latencies. For this descriptive analysis, the ERN latency was computed as the latency of the time point with the most negative amplitude within the $-300-600 \mathrm{~ms}$ time window. Two vertical green lines indicate the time window that was ultimately selected (-50 ms to $300 \mathrm{~ms})$.

\subsection{Statistical Analysis}

Our behavioural data analyses were focused on post-error go trials, whereas our ERP data analyses were performed on erroneous responses. We built three LME models: one to analyse the post-error slowing, one to analyse the ERN amplitudes, and one to analyse the ERN 
latencies. The models were fitted by restricted maximal likelihood (REML). Each LME model of ERP data had one random variable (participant); as fixed effects, each model included stop-response interval (as a variable of interest) and stop-signal delay (as a variable of no interest, which, however, may potentially explain the common variance with the stopresponse interval). The LME model of reaction latencies on post-error trials was constructed similarly, except that it did not contain stop-signal delay as a fixed effect. All models had a fully maximal random effects structure. The continuous predictors were centred and rescaled (divided by 100) prior to entering the analyses. Absolute t-values greater than the conventional level of 2 were considered significant. All analyses were conducted using the lme4 package for R (version 1.1-23; Bates et al., 2015).

In addition, we calculated the mean reaction times of correct go trials directly following unsuccessfully inhibited stop trials; we then compared them using the $t$-test with the mean reaction times of the correct go trials which were not preceded by stop trials. We also computed the stop-signal reaction time (only for descriptive purposes and following standard practice) as a behavioural variable unrelated to the research question. With this aim, reaction times from responses to go stimuli on which no stop-signal occurred were collapsed into a single distribution and rank ordered. The $n$th reaction time was selected, where $n$ was obtained by multiplying the number of no-signal reaction times in the distribution (150) by the probability of responding (e.g., 0.5 if the global inhibition rate was equal to 50\%) for each participant separately. Stop-signal reaction time was calculated by subtracting the average stop-signal delay from the $n$th reaction time, following the horse race model (see Logan $\&$ Cowan, 1984; Verbruggen \& Logan, 2008 for rationale). 
Significance of avoidable errors

\section{Results}

In the analysis of behavioural data, we mainly focused on post-error slowing. The mean reaction time of correct go trials that directly followed unsuccessfully inhibited stop trials was $431.8 \mathrm{~ms}(S D=76.0)$, whereas the mean reaction time of correct go trials which were not preceded by stop trials was $358.5 \mathrm{~ms}(S D=63.0)$. The difference between these two variables reveals the extent of post-error slowing, and it turned out to be statistically significant, $t(54)=11.24,95 \%$ CI: $60.26-86.42, p<.001, d=0.9$. To test our hypothesis concerning the relation between the mean reaction time of post-error go trials and the stopresponse interval in the previous trial, we built a linear mixed-effects (LME) model. The summary of results is shown in Table 1 (upper part). The analysis revealed a significant main effect of the stop-response interval in the previous trial, indicating that as the stop-response interval became longer, the post-error slowing increased in length. The overall behavioural performance was typical of the stop-signal task: the mean reaction time of correct go trials was $373.7 \mathrm{~ms}(S D=63.8)$; stop-signal delay was $160.8 \mathrm{~ms}(S D=47.6)$; stop-signal reaction time was $231.7 \mathrm{~ms}(S D=50.4)$.

Our ERP data analyses were focused on erroneous responses. We built two LME models: one to analyse the ERN amplitudes and one to analyse the ERN latencies. The results of the analyses of the mean amplitude and peak ERN latency are shown in Table 1 (middle and lower part, respectively). Both models revealed a significant main effect of the stopresponse interval. This indicates that as the stop-response interval became longer, the ERN increased and peaked earlier. To visualize these effects, Fig. 4 presents an ERP image of the ERN. In addition, both models showed that the effect of the stop-signal delay was not significant. 
Table 1 - The LME model's estimates of the mean reaction time of the post-error go trials (upper part), ERN amplitude (middle part) and latency (lower part). Random correlations are omitted.

\begin{tabular}{|c|c|c|c|c|}
\hline \multicolumn{5}{|c|}{ Mean reaction time of the post-error go trials } \\
\hline & & Tixed effects & & $\begin{array}{c}\text { Random effects } \\
\text { (by Subject) }\end{array}$ \\
\hline Effect & Estimate & $95 \% \mathrm{CI}$ & $t$-value & $S D$ \\
\hline Intercept & 432 & $412-453$ & 41.24 & 74 \\
\hline $\begin{array}{l}\text { Stop-response interval } \\
\text { in the previous trial }\end{array}$ & $\mathbf{0 . 1 9}$ & $0.11-0.28$ & 4.58 & 0 \\
\hline Residual & & & & 101 \\
\hline
\end{tabular}

\begin{tabular}{|c|c|c|c|c|}
\hline \multicolumn{5}{|l|}{ ERN amplitude } \\
\hline \multirow[b]{2}{*}{ Effect } & \multicolumn{3}{|c|}{ Fixed effects } & $\begin{array}{c}\text { Random effects } \\
\text { (by Subject) }\end{array}$ \\
\hline & Estimate & $95 \% \mathrm{CI}$ & $t$-value & $S D$ \\
\hline Intercept & -2.02 & $-3.33--0.72$ & -3.04 & 3.85 \\
\hline Stop-response interval & -2.22 & $-3.45--0.98$ & -3.51 & 2.93 \\
\hline Stop-signal delay & -0.24 & $-2.65-2.17$ & -0.20 & 6.84 \\
\hline Residual & & & & 11.26 \\
\hline \multicolumn{5}{|l|}{ ERN latency } \\
\hline & & Fixed effects & & $\begin{array}{c}\text { Random effects } \\
\text { (by Subject) }\end{array}$ \\
\hline Effect & Estimate & $95 \% \mathrm{CI}$ & $t$-value & $S D$ \\
\hline Intercept & 111.03 & $103.74-118.32$ & 29.85 & 18.68 \\
\hline Stop-response interval & -27.50 & $-34.95--20.05$ & -7.23 & 13.58 \\
\hline Stop-signal delay & 0.21 & $-11.84-12.26$ & 0.03 & 28.78 \\
\hline Residual & & & & 81.53 \\
\hline
\end{tabular}

Note: Significant effects are indicated in bold. 


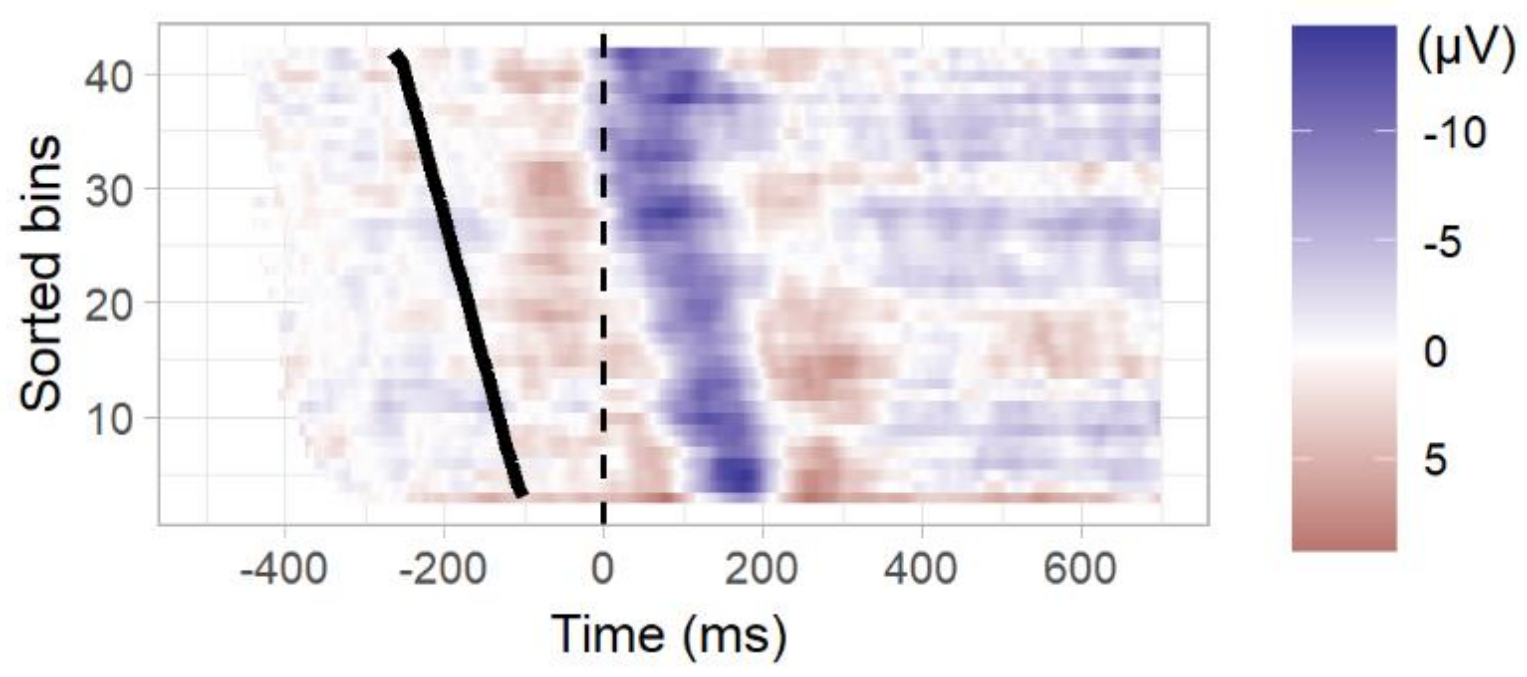

Fig. 4 - ERP image of the ERN effect. The trials were time-locked to the erroneous response and sorted as a function of stop-response interval (from the shortest to the longest stopresponse interval). The solid black line indicates the onset of the stop signal; the vertical dashed line indicates the onset of the error (point 0 on the horizontal axis). For illustrative purposes, single trials were aggregated into consecutive bins, as shown on the vertical axis. Each bin contained at least 10 single trials with exactly the same stop-response interval. Negative and positive amplitudes are depicted by a blue-to-red gradient.

\section{Discussion}

The aim of the present study was to test whether error-related brain responses which are associated with subjective evaluation of error significance are sensitive to the degree to which errors are avoidable, i.e. the degree to which humans can or cannot prevent themselves from committing errors. We hypothesized that the greater significance and higher motivational value of avoidable errors should be reflected in larger and earlier ERNs, and increased posterror slowing. To pursue our goal we used the stop-signal task, which is perfectly suited to eliciting inhibition errors that vary in the degree to which they can be avoided. As the index of error inevitability, we used the stop-response interval, i.e. the time between the presentation of 
the stop signal and the execution of the response. Prior research has shown that the smaller this distance, the less the error can be avoided. In particular, if the stop signal occurs after the so-called point of no return, the reaction-making process is so underway that it cannot be stopped (e.g., Schultze-Kraft et al., 2016). Consistent with our hypothesis, we observed that more avoidable errors (as indexed by the stop-response interval) are associated with larger and earlier ERNs and with increased post-error slowing, which is a behavioural index of error significance. Thus, the results show that early performance monitoring, as reflected by ERN, indeed involves an evaluation of error inevitability. In the following, we will discuss the results in more detail.

With regard to ERN latency, our analyses revealed that the longer the distance between the stop signal and the response, the earlier ERN reached its maximum. This finding suggests that avoidable errors take less time to be detected than unavoidable errors. In other words, if we have little time to withhold a response (because execution of the go response is already well under way), we need more time to detect an error; vice versa, if we have relatively more time to withhold a response, then we detect an error more quickly. This result allows a mechanistic explanation based on the dynamics of inhibitory processes. Inhibition of an erroneous response takes time to develop (e.g., Hasbroucq et al., 1999). In the stop-signal task, this process starts just after the presentation of the stop signal and may be described as a race between a 'go runner' and a 'stop runner', namely two opposing processes that are independent of each other (Logan \& Cowan, 1984). Since the stop signal is presented at varying intervals following the go stimulus, the inhibition may be initiated at different times after the go response is triggered: after the point of no return; when inhibition errors are unavoidable; or before the point of no return, when the likelihood of successful stopping is greater (cf., De Jong et al., 1990; Schultze-Kraft et al., 2016). In error trials in which the stop signal occurs after the point of no return, inhibitory processes do not have enough time to 
fully develop before the incorrect response is given. Therefore, the longer latency of the ERN peak in such trials may be due to the fact that the main part of the remedial action takes place after the reaction. In contrast, in error trials with a longer stop-response interval, the implementation of remedial action starts early enough to finish just after an erroneous response, thus triggering a short-latency ERN.

The most important finding of our study is that when the gap between the stop signal and the response was longer, i.e. when participants had more time to inhibit their response, the ERN amplitude was more pronounced. In the study by Vocat and colleagues (2011), ERN amplitude was strongly influenced by the magnitude of errors: ERN increased when the accuracy of ballistic pointing movements decreased, thus reflecting the severity of errors (see Bernstein et al., 1995; Luu et al., 2000 for similar examples). Our results confirm and extend these previous findings by demonstrating that ERN amplitude also varies with the degree to which errors are avoidable; this is consistent with the notion that the error-detection system is sensitive to the subjectively perceived significance and motivational value of an action failure (Hajcak et al., 2004; 2005; Maier et al., 2008). In other words, our results indicate that ERN amplitude codes for the significance of errors that are avoidable to a greater or lesser extent.

Another key result of our study is that the greater motivational value of avoidable errors was not only encoded in the neural indices of the error-detection mechanism but was also reflected on the behavioural level in increased post-error slowing. The pattern by which more significant errors, which produce larger ERNs, were associated with increased post-error slowing has been frequently reported in the literature (Debener et al., 2005; Holroyd et al., 2005; Yeung et al. 2004). Post-error slowing is typically assumed to reflect either adaptive cognitive control adjustments or automatic orientation of attention toward an unexpected event (for a review, see Danielmeier \& Ullsperger, 2011). In line with these explanations, our results indicate that more avoidable errors were either more able to trigger remedial action 
that was implemented in the form of cognitive control adjustments in order to reduce subsequent errors, or more unexpected for attentional orienting system than errors that could not have been avoided. Whatever the reason was, this pattern of results indicates the greater motivational influence of avoidable errors and their greater impact on subsequent behaviour.

In a broader perspective, our findings have implications for the attribution of moral responsibility. Rigoni and colleagues (Rigoni et al., 2012, 2013, 2015) observed that weakening belief in intentional control reduces behavioural and neural markers of performance monitoring and decreases the feeling of having deliberate control over a motor action (but see Eben et al., 2020). In other words, they showed that the basic neurocognitive processes that underlie voluntary behaviour are influenced by the extent to which people believe they can exert intentional control. Most importantly for the present study, the weaker the belief in free will, the less pronounced the ERN became (Rigoni et at., 2015). Our study went a step further by demonstrating that these basic neurocognitive processes are also affected by whether people are truly able to exert intentional control over their own behaviour. We observed that the greater the control over a motor action, the more pronounced and earlier the ERN.

Our results suggest that the attribution of moral responsibility for a given act, i.e. whether we are truly responsible for an action failure because we could have acted differently or are not responsible because we had no other choice, could be a well-elaborated reflection of how our brain initially classifies such a failure. The first assessment of error inevitability is performed at an early automatic stage of error processing, which is associated with subjective evaluation of error significance (as reflected by ERN). Naturally, in the present study we focused on extremely simple acts, i.e. motor responses generated in an artificial laboratory situation, which may limit the generalizability of our findings. In most everyday situations, attribution of moral responsibility refers to acts that are definitely more complex and requires 
Significance of avoidable errors

the evaluation of the impact of external and internal constraints. However, our results indicate that the elementary basis for distinguishing between culpable and non-culpable errors may occur in our brains several dozen milliseconds after error commission.

To conclude, when a car hits a pedestrian, we usually try to determine whether the pedestrian or the driver was at fault, and why the accident happened at all: because the pedestrian crossed the street just in front of the car, or because the driver applied the brakes too slowly? Although the damage caused by the driver could be similar in both scenarios, the significance of his act may be radically different. We aimed to determine whether this difference in error significance is detectable by our brains. We found that the higher motivational value of avoidable errors was indeed reflected in larger and earlier ERNs. Moreover, avoidable errors had a greater impact on future performance than errors that could not have been avoided. To sum up, at the beginning of our considerations we noted that according to the widely accepted moral principle, an agent is responsible for performing a given act only if he could have avoided performing it (Widerker \& McKenna, 2006). All indications suggest that the brain already realizes this in the first hundred milliseconds after the erroneous action.

\section{Competing interests}

The authors declare no competing interests.

\section{Open practices}

All data, scripts and supporting materials are available online at https://osf.io/hvj4s/

\section{Acknowledgments}

The authors are grateful to Michael Timberlake for proofreading the manuscript. 


\section{CrediT author statement}

Conceptualization: MS, JS; Data curation: MS, JS; Formal analysis: MS, JS; Investigation: MS; Visualization: MS, JS; Writing - original draft: MS, JS; Writing - review \& editing: MS, JS.

\section{References}

Bates, D., Mächler, M., Bolker, B., \& Walker, S. (2015). Fitting Linear Mixed-Effects Models using lme4. Journal of Statistical Software, 67, 1-48. https://doi.org/10.18637/jss.v067.i01

Bernstein, P. S., Scheffers, M. K., \& Coles, M. G. (1995). "Where did I go wrong?" A psychophysiological analysis of error detection. Journal of Experimental Psychology: Human Perception and Performance, 21, 1312-1322. https://doi.org/10.1037/0096-

\section{$\underline{1523.21 .6 .1312}$}

Danielmeier, C., \& Ullsperger, M. (2011). Post-error adjustments. Frontiers in Psychology, 2, 233. https://doi.org/10.3389/fpsyg.2011.00233

De Jong, R., Coles, M. G. H., Logan, G. D., \& Gratton, G. (1990). In search of the point of no return: The control of response processes. Journal of Experimental Psychology. Human Perception and Performance, 16, 164-182. https://doi.org/10.1037/0096-1523.16.1.164

Debener, S., Ullsperger, M., Siegel, M., Fiehler, K., Von Cramon, D. Y., \& Engel, A. K. (2005). Trial-by-trial coupling of concurrent electroencephalogram and functional magnetic resonance imaging identifies the dynamics of performance monitoring. Journal of Neuroscience, 25, 11730-11737. https://doi.org/10.1523/JNEUROSCI.3286-05.2005

Eben, C., Chen, Z., Cracco, E., Brass, M., Billieux, J., \& Verbruggen, F. (2020). Are posterror adjustments influenced by beliefs in free will? A failure to replicate Rigoni, Wilquin, 
Significance of avoidable errors

Brass and Burle, 2013. Royal Society Open Science, 7, 200664.

https://doi.org/10.1098/rsos.200664

Falkenstein, M., Hohnsbein, J., Hoormann, J., \& Blanke, L. (1990). Effects of errors in choice reaction tasks on the ERP under focused and divided attention. In C. H. M. Brunia, A. W.

K. Gaillard, A. Kok (Eds.), Psychophysiological brain research (Vol. 1, pp. 192-195).

Tilburg, The Netherlands: Tilburg University Press.

Ficarella, S. C., Rochet, N., \& Burle, B. (2019). Becoming aware of subliminal responses: an EEG/EMG study on partial error detection and correction in humans. Cortex, 120, 443 456. https://doi.org/10.1016/j.cortex.2019.07.007

Fiehler, K., Ullsperger, M., \& Von Cramon, D. Y. (2005). Electrophysiological correlates of error correction. Psychophysiology, 42, 72-82. https://doi.org/10.1111/j.1469$\underline{8986.2005 .00265 . x}$

Forster, K., \& Forster, J. C. (2003). DMDX: A Windows display program with millisecond accuracy. Behavior Research Methods, Instruments and Computers, 35, 116-124. https://doi.org/10.3758/BF03195503

Gehring, W. J., Goss, B., Coles, M. G. H., Meyer, D. E., \& Donchin, E. (1993). A neural system for error detection and compensation. Psychological Science, 4, 385-390. https://doi.org/10.1111/j.1467-9280.1993.tb00586.x

Hajcak, G., McDonald, N., \& Simons, R. F. (2004). Error-related psychophysiology and negative affect. Brain and Cognition, 56, 189-197. https://doi.org/10.1016/j.bandc.2003.11.001

Hajcak, G., Moser, J.S., Yeung, N., \& Simons, R.F. (2005). On the ERN and the significance of errors. Psychophysiology, 42, 151-160. https://doi.org/10.1111/j.1469$\underline{8986.2005 .00270 . x}$ 
Hasbroucq, T., Possamaï, C. A., Bonnet, M., \& Vidal, F. (1999). Effect of the irrelevant location of the response signal on choice reaction time: an electromyographic study in humans. Psychophysiology, 36, 522-526. https://doi.org/10.1017/S0048577299001602

Holroyd, C. B., Yeung, N., Coles, M. G., \& Cohen, J. D. (2005). A mechanism for error detection in speeded response time tasks. Journal of Experimental Psychology: General, 134, 163-191. https://doi.org/10.1037/0096-3445.134.2.163

Jung, T. P., Makeig, S., Humphries, C., Lee, T. W., Mckeown, M. J., Iragui, V., \& Sejnowski, T. J. (2000). Removing electroencephalographic artifacts by blind source separation. Psychophysiology, 37, 163-178. https://doi.org/10.1111/1469-8986.3720163

Kok, A., Ramautar, J. R., De Ruiter, M. B., Band, G. H., \& Ridderinkhof, K. R. (2004). ERP components associated with successful and unsuccessful stopping in a stop-signal task. Psychophysiology, 41, 9-20. https://doi.org/10.1046/j.1469-8986.2003.00127.x

Laming, D. (1979). Choice reaction performance following an error. Acta Psychologica, 43, 199-224. https://doi.org/10.1016/0001-6918(79)90026-X

Logan, G. D., \& Cowan, W. B. (1984). On the ability to inhibit thought and action: A theory of an act of control. Psychological Review, 91, 295-327. https://doi.org/10.1037/0033$\underline{295 X .91 .3 .295}$

Luu, P., Flaisch, T., \& Tucker, D. M. (2000). Medial frontal cortex in action monitoring. Journal of Neuroscience, 20, 464-469. https://doi.org/10.1523/JNEUROSCI.20-01$\underline{00464.2000}$

Maier, M., Steinhauser, M., \& Hübner, R. (2008). Is the error-related negativity amplitude related to error detectability? Evidence from effects of different error types. Journal of Cognitive Neuroscience, 20, 2263-2273. https://doi.org/10.1162/jocn.2008.20159

Miltner, W. H., Lemke, U., Weiss, T., Holroyd, C., Scheffers, M. K., \& Coles, M. G. (2003). Implementation of error-processing in the human anterior cingulate cortex: a source 
Significance of avoidable errors

analysis of the magnetic equivalent of the error-related negativity. Biological Psychology, 64, 157-166. https://doi.org/10.1016/S0301-0511(03)00107-8

Rabbitt, P. M. (1966). Errors and error correction in choice-response tasks. Journal of Experimental Psychology, 71, 264-272.

Ramautar, J. R., Kok, A., \& Ridderinkhof, K. R. (2004). Effects of stop-signal probability in the stop-signal paradigm: the N2/P3 complex further validated. Brain and Cognition, 56, 234-252. https://doi.org/10.1016/j.bandc.2004.07.002

Ridderinkhof, K. R., Ullsperger, M., Crone, E. A., \& Nieuwenhuis, S. (2004). The role of the medial frontal cortex in cognitive control. Science, 306, 443-447.

https://doi.org/10.1126/science.1100301

Rigoni, D., Kühn, S., Gaudino, G., Sartori, G., \& Brass, M. (2012). Reducing self-control by weakening belief in free will. Consciousness and Cognition, 21, 1482-1490. https://doi.org/10.1016/j.concog.2012.04.004

Rigoni, D., Pourtois, G., \& Brass, M. (2015). 'Why should I care?'Challenging free will attenuates neural reaction to errors. Social Cognitive and Affective Neuroscience, 10, 262268. https://doi.org/10.1093/scan/nsu068

Rigoni, D., Wilquin, H., Brass, M., \& Burle, B. (2013). When errors do not matter: Weakening belief in intentional control impairs cognitive reaction to errors. Cognition, 127, 264-269. https://doi.org/10.1016/j.cognition.2013.01.009

Roger, C., Núñez Castellar, E., Pourtois, G., \& Fias, W. (2014). Changing your mind before it is too late: the electrophysiological correlates of online error correction during response selection. Psychophysiology, 51, 746-760. https://doi.org/10.1111/psyp.12224

Schevernels, H., Bombeke, K., Van der Borght, L., Hopf, J. M., Krebs, R. M., \& Boehler, C. N. (2015). Electrophysiological evidence for the involvement of proactive and reactive 
Significance of avoidable errors

control in a rewarded stop-signal task. NeuroImage, 121, 115-125.

https://doi.org/10.1016/j.neuroimage.2015.07.023

Schultze-Kraft, M., Birman, D., Rusconi, M., Allefeld, C., Görgen, K., Dähne, S., ... \&

Haynes, J. D. (2016). The point of no return in vetoing self-initiated movements.

Proceedings of the National Academy of Sciences, 113, 1080-1085.

https://doi.org/10.1073/pnas.1513569112

Senderecka, M., Szewczyk, J., Wichary, S., \& Kossowska, M. (2018). Individual differences in decisiveness: ERP correlates of response inhibition and error monitoring.

Psychophysiology, 55, e13198. https://doi.org/10.1111/psyp.13198

Verbruggen, F. \& Logan, G. (2008). Response inhibition in the stop-signal paradigm. Trends in Cognitive Sciences, 12, 418-424. https://doi.org/10.1016/j.tics.2008.07.005

Vocat, R., Pourtois, G., \& Vuilleumier, P. (2011). Parametric modulation of error-related ERP components by the magnitude of visuo-motor mismatch. Neuropsychologia, 49, 360-367. https://doi.org/10.1016/j.neuropsychologia.2010.12.027

Widerker, D., \& McKenna, M. (2006). Moral responsibility and alternative possibilities:

Essays on the importance of alternative possibilities. Aldershot, UK: Ashgate.

World Medical Organization. (1996). Declaration of Helsinki. British Medical Journal, 313, $1448-1449$.

Yeung, N., Botvinick, M. M., \& Cohen, J. D. (2004). The neural basis of error detection: Conflict monitoring and the error-related negativity. Psychological Review, 111, 931-959. https://doi.org/10.1037/0033-295X.111.4.931 